\title{
Transient mesoportal shunt: An innovative technique for maintaining portal flow during pancreatoduodenectomy with portal vein resection
}

\author{
Gautham M. S., Senthil Gnanasekaran, Kalayarasan Raja, and Biju Pottakkat \\ Department of Surgical Gastroenterology, Jawaharlal Institute of \\ Postgraduate Medical Education and Research (JIPMER), Puducherry, India
}

\begin{abstract}
Pancreatic cancers exhibit a surgical challenge, in light of frequent vascular involvement. In absence of metastatic spread, vascular invasion is the predominant limiting factor for determining the resectability. With progression of time vascular involvement is no longer considered a surgical contraindication. However these complex procedures are fraught with technical challenges. Portal clamping required for vascular resection and reconstruction results in hepatic ischemia and visceral congestion. In order to mitigate these untoward effects, surgeons have tried diverse techniques including venous shunts. Venous shunting facilitates the resection and allows for an enhanced exposure and a safe procedure. Previously described techniques were either cumbersome or failed to maintain portal flow. We present a technique of transient mesoportal shunt, to facilitate vascular resection during pancreatoduodenectomy. This technique is both simple and maintains portal flow throughout the procedure preventing both hepatic ischemia and visceral congestion. (Ann Hepatobiliary Pancreat Surg 2021;25:122-125)
\end{abstract}

Key Words: Borderline resectable carcinoma pancreas; Transient mesoportal shunt; Pancreatoduodenectomy; Vascular resection

\section{INTRODUCTION}

Surgical resection provides only chance of cure in patients with pancreatic malignancy. Unfortunately less than $20 \%$ of patients have resectable disease at presentation. With acquisition of greater skills in pancreatic surgery and improvement in perioperative care, the morbidity and mortality of pancreatoduodenectomy (PD) have significantly decreased in recent times. Hence, complex PD with combined vascular resection are more frequently performed now-a-days without significant increase in postoperative morbidity and mortality. ${ }^{1}$ However, the concern regarding the deleterious effect of portal vein clamping on liver function and bowel congestion still remains. Portosystemic shunts have been used to circumvent these effects; however they only focused on maintaining the mesenteric flow. We describe a transient mesoportal shunting techni- que, used for vascular resection in a patient with borderline resectable pancreatic malignancy. The mesenteric flow was effectively bypassed to the portal vein, hence decompressing the mesenteric system and simultaneously maintaining the liver blood flow.

\section{CASE REPORT}

The patient is a 65 year old recently diagnosed diabetic, presented with features of malignant obstructive jaundice for 1 month. He was diagnosed with a pancreatic head lesion after ultrasonography. The serum bilirubin was 23 $\mathrm{mg} / \mathrm{dl}$ and CA 19-9 was elevated (263 IU/ml). Pancreatic phase protocol CT revealed a lesion in pancreatic head and uncinate region with $>180$ degree involvement of superior mesenteric vein-portal vein (SMV-PV) confluence. The length of involvement was about $3 \mathrm{~cm}$, with contour

Received: February 13, 2020; Revised: August 10, 2020; Accepted: August 28, 2020

Corresponding author: Senthil Gnanasekaran

Department of Surgical Gastroenterology, Jawaharlal Institute of Postgraduate Medical Education and Research (JIPMER), Puducherry 605006, India

Tel: +91-7094640190, Fax: +91-413-229, E-mail: senthilg8303@gmail.com

Copyright (C) 2021 by The Korean Association of Hepato-Biliary-Pancreatic Surgery

This is an Open Access article distributed under the terms of the Creative Commons Attribution Non-Commercial License (http://creativecommons.org/ licenses/by-nc/4.0) which permits unrestricted non-commercial use, distribution, and reproduction in any medium, provided the original work is properly cited. Annals of Hepato-Biliary-Pancreatic Surgery • pISSN: 2508-5778 - eISSN: 2508-5859 
abnormality (Fig. 1). The lesion was also abutting common hepatic artery (CHA) for a short length. Preoperative biliary drainage with endobiliary stent was done in view of very high bilirubin and uncontrolled diabetes mellitus. Subsequently surgery was performed after optimization of blood sugars and at serum bilirubin level of $9 \mathrm{mg} / \mathrm{dl}$. Diagnostic laparoscopy was done to rule out occult metastatic disease. The patient underwent PD with an artery first approach. Transient mesoportal shunt (TMPS) was constructed to facilitate portal vein resection anastomosis, in view of anticipated prolonged duration for vascular reconstruction, and to facilitate the retropancreatic dissection.

\section{TECHNIQUE DESCRIPTION}

Complete exposure was achieved by the Catell-Braasch maneuver. A posterior artery first approach was adopted and superior mesenteric artery (SMA) was cleared from its take off at aorta. A hard tumor was noted in the head and uncinate region of pancreas, which was infiltrating SMV-PV confluence. The Tumor was abutting CHA, but free from SMA and Celiac axis (Fig. 2).

With meticulous dissection, hilar PV and SMV were skeletonized. Unfractionated heparin was administered and side-to-side TMPS was constructed from SMV to hilar PV with a $10 \mathrm{~mm}$-Dacron graft of $15 \mathrm{~cm}$ length, using side biting vascular clamps (Fig. 3). Segment of PV-
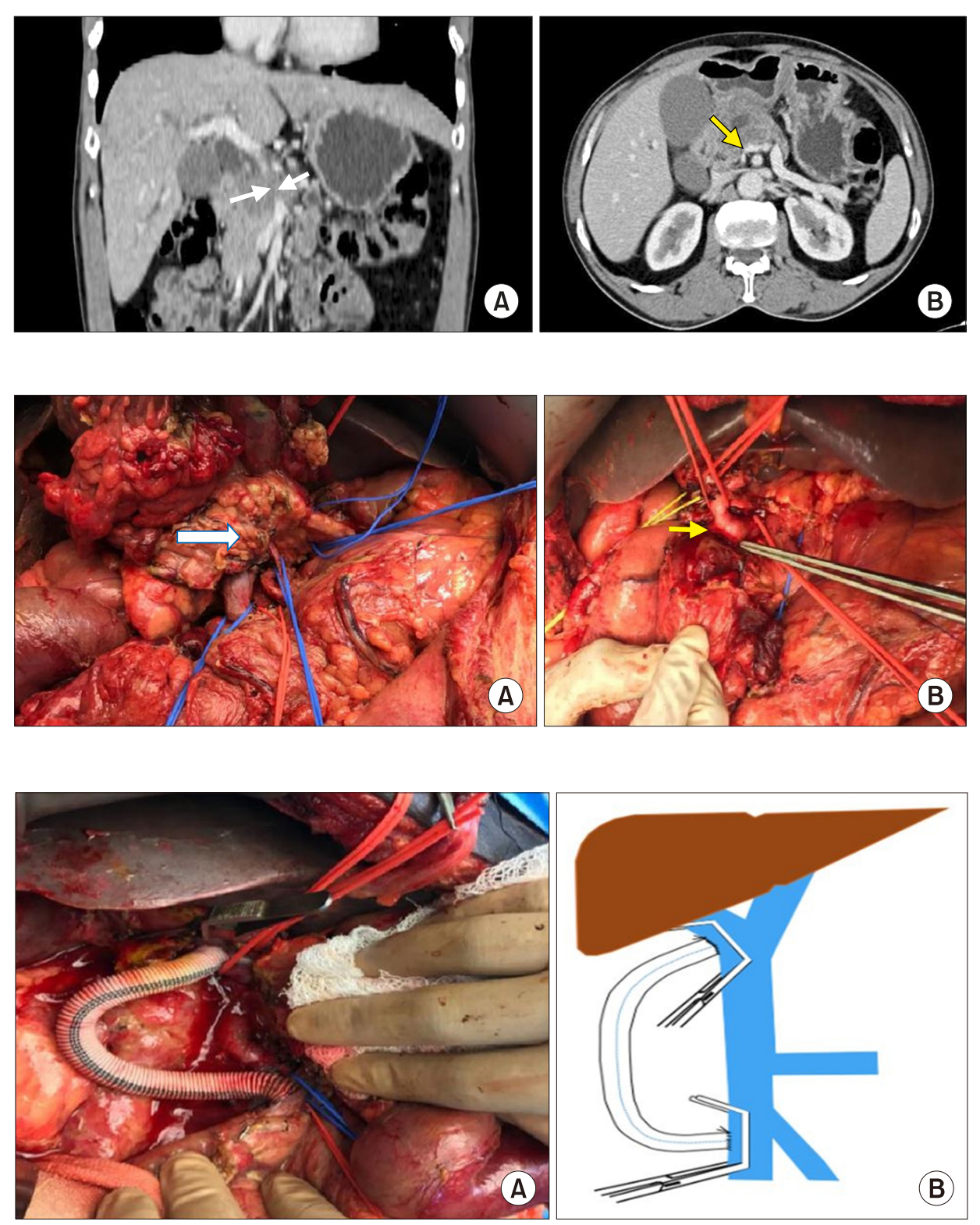

Fig. 3. Intraoperative images demonstrating construction of TMPS. (A) TMPS constructed after application of side biting vascular clamps, with a $10 \mathrm{~mm}$ Dacron graft. (B) Schema depicting the construction of TMPS. 

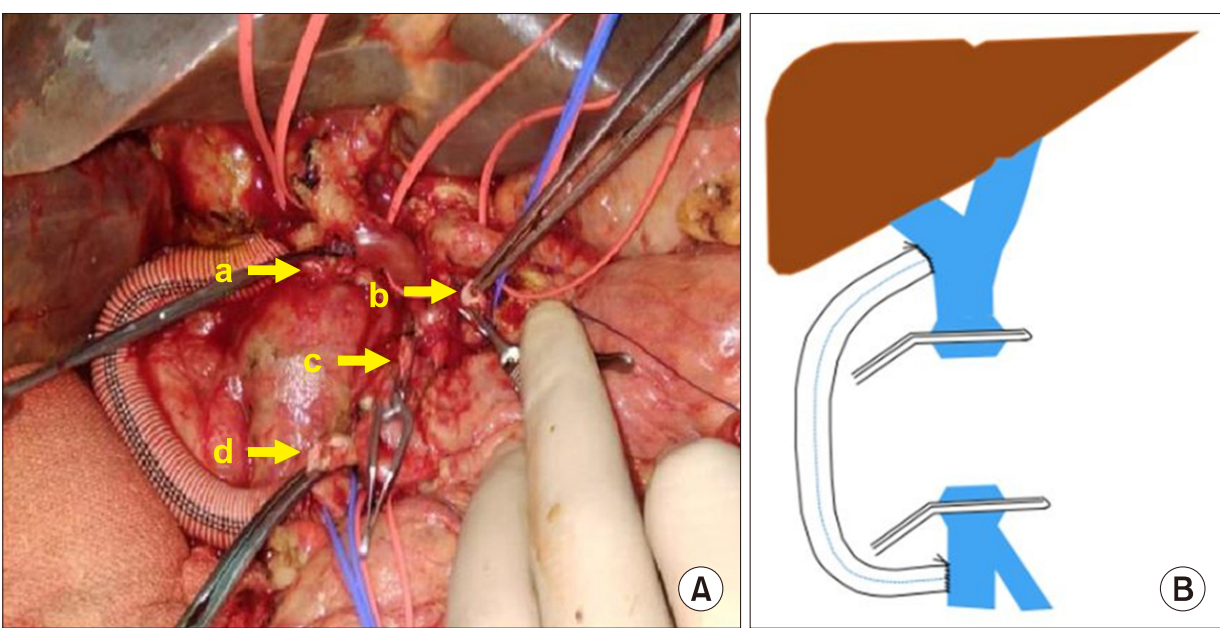

Fig. 4. Intraoperative images showing tumor bed. (A) Tumor was resected enbloc with a segment of PV-SMV confluence. Arrows depict transected ends of portal vein (a), splenic vein (b), IMV (c), SMV (d). (B) Schema showing operative field after enbloc resection.
SMV along with confluence was resected enbloc with the tumor (Fig. 4).

An end-to-end anastomosis of PV-SMV was done after adequate liver mobilization. Splenic vein flow was shunted to IMV. Duct-to-mucosa pancreatojejunostomy, hepaticojejunostomy and gastrojejunostomy were done in a usual manner. Total duration of the surgery was $420 \mathrm{mi}-$ nutes and blood loss was $300 \mathrm{ml}$. Postoperative recovery was uneventful, without any significant derangement of liver function test. Patient was discharged in a stable condition on $9^{\text {th }}$ postoperative day without any complications. Histopathology revealed a R0 resection. He received adjuvant chemotherapy and there was no evidence of tumor recurrence at 16 months of follow up after surgery.

\section{DISCUSSION}

The concept of vascular resection for pancreatic malignancy has evolved from routine regional pancreatectomy to enbloc resection in selected patients. ${ }^{2,3}$ Portal vein involvement is no longer considered either a technical or oncological contraindication for resection, provided a R0 resection can be achieved safely. Portal vein resection provides opportunity for many patients to undergo resection, which were traditionally considered unresectable. However, these procedures often require complex vascular resection and reconstruction. Intraoperative blood loss from the collaterals, visceral congestion and hepatic ischemia are the main concerns during these procedures. It has been proposed that portal vein clamping can be safely performed if reconstruction time is less than 30 minutes, and mesentericosystemic shunt is required if the time exceeds 60 minutes. ${ }^{4}$ However this may not be applicable universally, as the physiologic effects of portal clamping during PD depends on many factors including functional status of the liver. Hence, various venous shunting procedures have been adopted by surgeons.

The mesocaval shunt typically aims at reducing the collateral flow, secondary to portal vein obstruction. This helps in reducing the blood loss especially in Nakao type $\mathrm{C}$ and D type portal vein involvement. The use of mesocaval and distal splenorenal shunt has been described in literature for this purpose, without major postoperative complications. $^{5,6}$

The mesocaval shunt effectively decompresses the mesenteric venous system, and the collateral flow. However, the concern with this technique is the complete diversion of portal flow away from the liver. Interruption of the portal flow even for a short duration may have deleterious effects in the presence of cholestasis or preoperative chemotherapy related liver injury.

Nakao et al. ${ }^{7}$ used an antithrombogenic heparin coated temporary catheter to construct a mesoportal shunt between SMV and either hilar PV or umbilical vein in various hepatopancreatobiliary conditions, requiring vascular resections. A more cumbersome procedure with use of centrifugal pump-assisted bypass has also been described. ${ }^{8}$ Bachellier et al. ${ }^{9}$ have reported the use of Gore-Tex graft for a temporary end-to-end mesoportal shunt in Nakao type $\mathrm{C}$ and $\mathrm{D}$ patients. However, this technique requires interruption of portal flow while shunting, as well as during reconstruction of portal vein. 
We used a readily available Dacron graft with standard vascular anastomotic techniques to create TMPS. The procedure is simple, requires standard vascular technique, which is easily reproducible and does not require centrifugal pumps or other cumbersome equipment. The present techniques allow uninterrupted portal flow during construction of the shunt, resection of SMV-PV confluence and its reconstruction. Hence, hepatic ischemia and bowel congestion due to prolonged portal vein clamping can be prevented. The SMA clamping, which is done to reduce bowel congestion during portal flow interruption can be completely avoided, thus preventing its associated reperfusion effects. The temporary side-to-side shunt reduces the time constraints of prolonged clamping, optimizes the surgeon's ability to achieve a R0 resection and facilitates the retropancreatic dissection. Hence, this technique can be used in borderline resectable pancreatic malignancies that closely abut the SMA or involve the CHA, requiring prolonged Portal vein clamping and complex vascular reconstruction.

\section{CONCLUSION}

Transient mesoportal shunt (TMPS) using Dacron graft is an easy and effective strategy to decompress the mesenteric venous system, and to maintain the portal flow during complex portal vein resection and anastomosis in pancreatoduodenectomy.

\section{ACKNOWLEDGEMENTS}

We thank Dr. Satyaprakash Ray Choudhury for his useful suggestions.

\section{CONFLICT OF INTEREST}

The authors declare that they have no competing interest.

\section{ORCID}

Gautham M. S.: https://orcid.org/0000-0002-6636-1648

Senthil Gnanasekaran: https://orcid.org/0000-0002-8639-5423

Kalayarasan Raja: https://orcid.org/0000-0003-4056-8672

Biju Pottakkat: https://orcid.org/0000-0002-8474-0270

\section{AUTHOR CONTRIBUTIONS}

Conceptualization: SG. Writing - original draft: GMS. Writing - review \& editing: SG, KR, BP.

\section{REFERENCES}

1. Beane JD, House MG, Pitt SC, Zarzaur B, Kilbane EM, Hall $\mathrm{BL}$, et al. Pancreatoduodenectomy with venous or arterial resection: a NSQIP propensity score analysis. HPB (Oxford) 2017;1 9:254-263.

2. Fortner JG. Regional pancreatectomy for cancer of the pancreas, ampulla, and other related sites. Tumor staging and results. Ann Surg 1984;199:418-425.

3. Asada S, Itaya H, Nakamura K, Isohashi T, Masuoka S. Radical pancreatoduodenectomy and portal vein resection. Report of two successful cases with transplantation of portal vein. Arch Surg 1963;87:609-613.

4. Tashiro S, Uchino R, Hiraoka T, Tsuji T, Kawamoto S, Saitoh $\mathrm{N}$, et al. Surgical indication and significance of portal vein resection in biliary and pancreatic cancer. Surgery 1991;109:481487.

5. Christians KK, Riggle K, Keim R, Pappas S, Tsai S, Ritch P, et al. Distal splenorenal and temporary mesocaval shunting at the time of pancreatectomy for cancer: initial experience from the Medical College of Wisconsin. Surgery 2013;154:123-131.

6. Pilgrim CH, Tsai S, Evans DB, Christians KK. Mesocaval shunting: a novel technique to facilitate venous resection and reconstruction and enhance exposure of the superior mesenteric and celiac arteries during pancreaticoduodenectomy. J Am Coll Surg 2013;217:e17-e20.

7. Nakao A, Nonami T, Harada A, Kasuga T, Takagi H. Portal vein resection with a new antithrombogenic catheter. Surgery 1990; 108:913-918.

8. Suzuki Y, Yoshida I, Ku Y, Fujino Y, Tanioka Y, Fukumoto $\mathrm{T}$, et al. Safety of portal vein resection using centrifugal pumpassisted venous bypass between the superior mesenteric vein and the umbilical vein. Hepatogastroenterology 2004;51:1183-1186.

9. Bachellier P, Rosso E, Fuchshuber P, Addeo P, David P, Oussoultzoglou E, et al. Use of a temporary intraoperative mesentericoportal shunt for pancreatic resection for locally advanced pancreatic cancer with portal vein occlusion and portal hypertension. Surgery 2014;155:449-456. 